\title{
The application of a VUV Fourier transform spectrometer and synchrotron radiation source to measurements of: II. The $\delta(1,0)$ band of NO
}

\author{
T. Imajo \\ Kyushu University, Fukuoka 812, Japan \\ K. Yoshino, J. R. Esmond, and W. H. Parkinson \\ Harvard-Smithsonian Center for Astrophysics, Cambridge, Massachusetts 02138
}

A. P. Thorne, J. E. Murray, R. C. M. Learner, and G. Cox

Blackett Laboratory, Imperial College, London SW7-2BZ, United Kingdom

\author{
A. S.-C. Cheung \\ The University of Hong Kong, Hong Kong \\ K. Ito and T. Matsui ${ }^{\mathrm{a})}$ \\ Photon Factory, KEK, Tsukuba, Ibaraki 305, Japan
}

(Received 7 October 1999; accepted 4 November 1999)

\begin{abstract}
Line-by-line photoabsorption cross-sections of the NO $\delta(1,0)$ band were measured with the VUV Fourier transform spectrometer from Imperial College, London, using synchrotron radiation at Photon Factory, KEK, Japan, as a continuum light source. The analysis of the NO $\delta(1,0)$ band provides accurate rotational line positions and term values as well as the photoabsorption cross-sections. The molecular constants of the $C(1)^{2} \Pi$ level are found to be $T_{0}=54690.155$ $\pm 0.03 \mathrm{~cm}^{-1}, \quad B_{\mathrm{v}}=1.94406 \pm 0.00062 \mathrm{~cm}^{-1}, \quad D_{\mathrm{v}}=(5.91 \pm 0.42) \times 10^{-5} \mathrm{~cm}^{-1}, \quad A_{D}=-0.0187$ $\pm 0.0050 \mathrm{~cm}^{-1}, p=-0.0189 \pm 0.0037 \mathrm{~cm}^{-1}$, and $q=-0.01521 \pm 0.00020 \mathrm{~cm}^{-1}$. The sum of the line strengths for all rotational transitions of the NO $\delta(1,0)$ band is determined as 4.80 $\times 10^{-15} \mathrm{~cm}^{2} \mathrm{~cm}^{-1}$, corresponding to a band oscillator strength of $0.0054 \pm 0.0003$. (C) 2000 American Institute of Physics. [S0021-9606(00)00805-9]
\end{abstract}

\section{INTRODUCTION}

Molecular absorption of ultraviolet solar radiation in the middle atmosphere has been discussed by a number of authors. $^{1-4}$ In the wavelength region $175-205 \mathrm{~nm}$ the penetration of solar radiation into the atmosphere is controlled by the absorption cross-sections of the Schumann-Runge (SR) bands of $\mathrm{O}_{2}$. Part of the radiation transmitted is available to photopredissociate NO, which has a number of strong bands with very narrow lines in the region 183-195 nm. The photodestruction of $\mathrm{O}_{2}$ and $\mathrm{NO}$ is recognized as important stratospheric processes, and their evaluation requires accurate knowledge of the rotational structure of the band systems. For accurate modeling of the processes the computations must be performed on a line-by-line basis: photoabsorption cross-sections, measured in the laboratory with resolution sufficient to yield true cross-sections, are required for both molecules. For most of the SR bands of ${ }^{16} \mathrm{O}_{2}$ such high resolution measurements have been completed and the results incorporated in models of $\mathrm{O}_{2}$ transmissions and photolysis (e.g., Nicolet and Kennis, ${ }^{5}$ Minschwaner et al. ${ }^{6}$ ).

The NO lines are considerably sharper than the $\mathrm{O}_{2} \mathrm{SR}$ lines. Published integrated cross-sections ${ }^{7-9}$ measured at insufficient spectral resolution in some cases differ by more than $50 \%$; the differences could indicate that some of the measurements were plagued by saturation, which is very difficult to detect when linewidths are much narrower than the

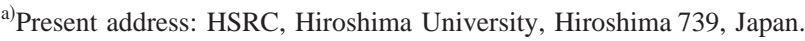

instrument widths. ${ }^{10}$ To overcome the problems of insufficient resolution, line-by-line measurements of the absorption cross-sections of the $\delta(0,0)$ and $\beta(7,0)$ bands of NO in the region $195-183 \mathrm{~nm}$ were performed by Murray et al. ${ }^{11}$ using the Fourier transform (FT) spectrometers at Imperial College, London, (IC) with a resolution of $0.06 \mathrm{~cm}^{-1}$. A positive-column hydrogen discharge at high current $(\sim 500 \mathrm{~mA})$ was used for a background continuum, with a $0.3 \mathrm{~m}$ Czerny-Turner spectrometer (McPherson model 218) to limit the bandwidth to 1 to $2 \mathrm{~nm}$. A signal-to-noise $(\mathrm{S} / \mathrm{N})$ ratio of 25 was achieved by coadding up to 128 runs, but this was not sufficient to determine the photoabsorption crosssections of weaker lines accurately. Because the signal-tonoise ratio achieved was close to the expected photon noise limit, it could be significantly improved only by using a brighter background continuum light source, such as synchrotron radiation.

Recently we combined high-resolution VUV Fourier transform spectrometry with synchrotron radiation by taking the IC VUV FT spectrometer, to the synchrotron radiation source at Photon Factory, KEK, Japan, where a suitable zero dispersion two-grating predisperser is available on beam line 12-B. (The predisperser is necessary to limit the bandwidth to a few $\mathrm{nm}$ in order to achieve acceptable $\mathrm{S} / \mathrm{N}$ ratios.) $\mathrm{We}$ have used this combination of facilities to make ultra high resolution cross-section measurements of $\mathrm{NO}$ and $\mathrm{O}_{2}$ bands in the wavelength region $195-160 \mathrm{~nm}$. Results for the $\beta(9,0)$ $\left(B^{2} \Pi_{r}-X^{2} \Pi_{r}\right)$ band of NO have recently been reported by 
Yoshino et al. ${ }^{12}$ The present article presents the spectroscopic analysis and the line and band oscillator strengths of the $\delta(1,0)\left(C^{2} \Pi_{r}-X^{2} \Pi_{r}\right)$ band of NO.

The close coincidence of the $\mathrm{v}=0$ level of $C(\mathrm{v})^{2} \Pi$ with the first dissociation energy of NO results in predissociation by interaction with the $a^{4} \Pi$ continuum. ${ }^{13,14}$ Predissociation has been confirmed by radiative lifetime measurements of the $C(0)$ rotational levels: a sudden change of the lifetime of the rotational levels was measured, from around $25 \mathrm{~ns}$ for low $J$ to $1 \mathrm{~ns}$ for high $J .^{15-17}$ Attempts have also been made to measure the lifetime of the $C(1)$ level, which is very short, in the range of 0.03 to $0.4 \mathrm{~ns} .^{15,18,19}$ Amiot and Verges ${ }^{20}$ performed emission studies of the $D-A$ and $C-A$ bands at high resolution, $0.007 \mathrm{~cm}^{-1}$, with an infrared Fourier transform (IR FT) spectrometer. They observed the $C(1)-A$ (1) band for the first time in emission, with broad rotational lines indicating predissociation of the $C(1)$ level. The intensity and linewidth studies have not been published. $^{21}$

The cross-section measurements of the $C(1)-X(0)$ band by Bethke $^{7}$ were performed at low resolution, $0.04 \mathrm{~nm}$, in the presence of Ar. The rotational lines were pressurebroadened to well beyond the instrumental width, enabling the true cross-sections to be measured. Callear and Pilling ${ }^{13}$ suggested that the results for the delta bands might be in error because the collision broadening is different for lines that are predissociated and lines that are not. $\mathrm{Cieslik}^{8}$ made low resolution measurements of NO at low pressure and used the equivalent width and curve of growth to obtain the band oscillator strength of the $\delta(1,0)$ band and obtained a result in good agreement with Bethke's. Guest and Lee ${ }^{9}$ used a synchrotron source and resolution of $0.03 \mathrm{~nm}$ with very low pressures of NO $\left(2 \times 10^{-4}\right.$ Torr $)$. Their result for the $\delta(1,0)$ band is $48 \%$ higher than Bethke's. Chan et al. ${ }^{22}$ used the high resolution dipole $(e, e)$ technique, which is not sensitive to the instrumental resolution. Their value of the oscillator strength of the $\delta(1,0)$ band agrees with Bethke's.

\section{EXPERIMENT}

Details of the experimental setup were described in our previous article. ${ }^{12}$ The synchrotron radiation transmitted by the zero-dispersion predisperser in the 12-B beam line of the Photon Factory was reflected by a cylindrical mirror and focused onto the entrance aperture of the FT spectrometer. The center wavelength and bandwidth of the transmitted radiation were set at 185 and $2 \mathrm{~nm}$, respectively to cover the $\delta(1,0)$ band of NO. The IC VUV spectrometer is equipped with a beam splitter made from a single crystal of $\mathrm{MgF}_{2} .{ }^{23}$ The spectral resolution of the FT spectrometer was $0.06 \mathrm{~cm}^{-1}$, requiring an entrance aperture of $1.5 \mathrm{~mm}$ diameter. The absorption cell, with an optical path length of $0.565 \mathrm{~cm}$, was placed between the predisperser and the FT spectrometer, and NO gas was introduced at a pressure of 0.4 Torr, corresponding to a column density of $7.40 \times 10^{15} \mathrm{~mol} \mathrm{~cm}^{-2}$. The NO pressure was measured with a capacitance manometer (MKS Baratron). In this case we coadded 320 scans, corresponding to about $14 \mathrm{~h}$ integration time. The $\mathrm{S} / \mathrm{N}$ ratio in the continuum background was 96. Another spectrum was recorded at the same optical path length with a different NO pressure, 0.2 Torr, in order to estimate the error in pressure measurements. For this spectrum we coadded 160 scans, and the $\mathrm{S} / \mathrm{N}$ ratio in the continuum background was 59 . In addition, a measurement with an optical path length of $0.28 \mathrm{~cm}$ and NO pressure of 0.314 Torr was made, yielding a $\mathrm{S} / \mathrm{N}$ ratio of 72 after coadding 224 scans. The column densities of these two measurements were $3.70 \times 10^{15}$ and 2.92 $\times 10^{15} \mathrm{~mol} \mathrm{~cm}^{-2}$, respectively.

\section{ANALYSIS OF THE SPECTRUM}

\section{A. Fitting of the FTS data}

The transmission spectra were converted to optical depth by taking the logarithms of the intensity and fitting a smooth continuum to the regions between the lines. The absorption lines were fitted to Voigt profiles using the spectral reduction routine GREMLIN. ${ }^{24}$ Line parameters are determined through a nonlinear least-squares iterative procedure. The Voigt profile for the NO lines should be a convolution of a Gaussian due to Doppler broadening and a Lorentzian arising from predissociation. In this case the Gaussian component of the best fit Voigt function had a full width at half maximum (FWHM) of $0.175 \mathrm{~cm}^{-1}$ which is significantly larger than the value of $0.12 \mathrm{~cm}^{-1}$ expected for Doppler width in our experimental conditions. This anomalous Gaussian width is considered to be due to drifts in alignment causing very small wave number shifts over the long observation periods. ${ }^{25}$

\section{B. Rotational term values of the $C(1)$ level}

The $\delta(1,0)$ band is shown in Fig. 1(a), and a portion of the spectrum is expanded in Fig. 1(b) to show the detail of the rotational structure. We observed 201 lines, and these lines are assigned in Table I to 235 rovibronic transition of $C^{2} \Pi(\mathrm{v}=1)-X^{2} \Pi(\mathrm{v}=0)$ according to the results of Lagerqvist and Miescher. ${ }^{26}$ Three lines of the $Q_{11}(J)$ and $Q_{22}(J)$ branches were assigned in the present work for the first time. Although the majority of the lines are separated, about onethird of them are blended with lines from other branches or from the same branch with a different $J$ quantum number. These are marked with $\mathrm{a} B$ or $\mathrm{b}$ following the wave numbers in the table. Blended lines followed by B were treated as a single line, while line positions followed by $b$ were extracted by a deconvolution procedure for an incompletely resolved complex. At least one reference wave number is needed for absolute calibration in FT spectroscopy. The resonance line of $\mathrm{Hg} \mathrm{I}$ at $184.8 \mathrm{~nm}$ was used as reference wave number in our previous article. ${ }^{12}$ In this wavelength region we have no convenient absolute reference. However, there is a small overlap between the two spectra, from which we can derive a calibration constant for the present spectrum with an uncertainty of $0.02 \mathrm{~cm}^{-1}$. Relative uncertainties for the strong lines are better than $0.01 \mathrm{~cm}^{-1}$. Differences in the observed line positions between our results and Lagerqvist and Miescher ${ }^{26}$ are plotted in Fig. 2. They claimed an uncertainty in their line positions of $0.1 \mathrm{~cm}^{-1}$ which is consistent with the results shown in Fig. 2.

The rotational term values of the $v=1$ level of the $C^{2} \Pi_{r}$ state can be obtained by adding the term values of the $X(0)$ levels to the wave numbers of the observed lines. The 

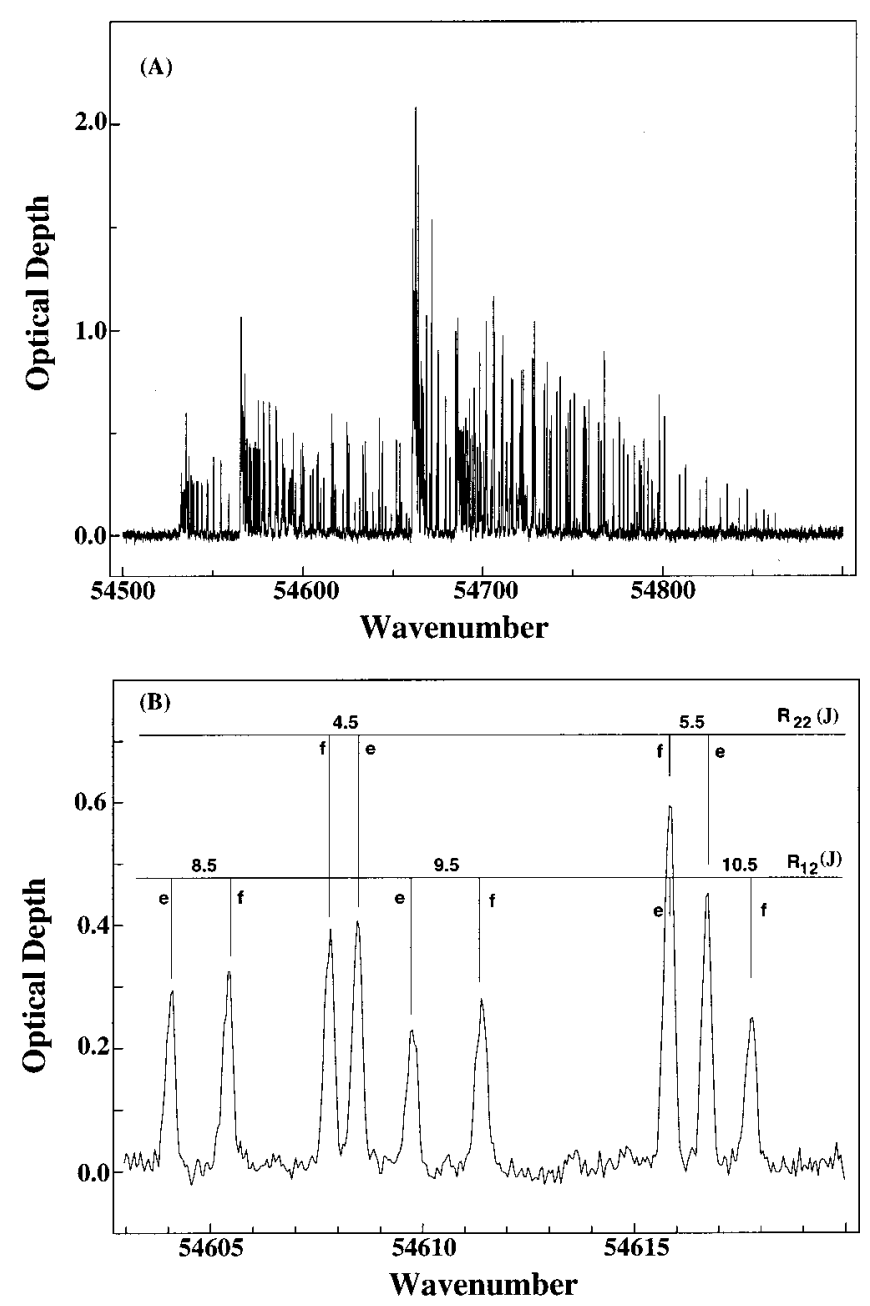

FIG. 1. The $\delta(1,0)$ band at 0.400 Torr of NO with $0.565 \mathrm{~cm}$ pathlength is shown in Fig. 1(a). A portion of the spectrum is expanded in Fig. 1(b). Separations of the $\Lambda$-type doubling are seen in $R_{12}$ and $R_{22}$ branch lines.

rotational term values of the $X(0)$ levels relative to the level $\Omega=\frac{1}{2}, J=0.5, e$ are given by Amiot $e t a l^{27}$ Our term values are obtained by averaging over the branch lines. The term values recorded from the three spectra at different column densities coincided within $0.05 \mathrm{~cm}^{-1}$. The average term values from the three runs are listed in Table II, with the standard deviation shown as an estimated error. The uncertainty in the absolute value of $T_{0}$ including the calibration uncertainty is $0.03 \mathrm{~cm}^{-1}$.

The effective Hamiltonian operator, suitable for the description of the ${ }^{2} \Pi$ states, can be found in Hougen ${ }^{28}$ and Zare et al. ${ }^{29}$ The matrix elements used in this work for the calculation of rotational energy levels are the same as those in Amiot et al..$^{30}$ and Stark et al. ${ }^{31}$ The parameters included in the description of the ${ }^{2} \Pi$ state are: The band origin $T_{0}$; the rotational parameters $B$ and $D$; the spin-orbit parameters $A$ and $A_{D}$; and the $\Lambda$-doubling parameters $p$ and $q$. In our analysis, we have performed least-squares fittings to the rovibronic term values. The term values corresponding to $J$ values above 11.5 were not included in the fit because line shifts were expected from perturbation by the $B(10)$ level. Table II summarizes the molecular constants obtained for the $\mathrm{v}=1$ level of the $C^{2} \Pi$ state.

\section{The integrated cross-sections of the $\delta(1,0)$ band}

As mentioned before, the absorption lines are fitted to Voigt profiles while the Gaussian contribution is kept constant as $0.175 \mathrm{~cm}^{-1}$. The fitting procedure provides integrated cross-sections as well as line positions. These are independent of the anomalous widths, provided that the residuals from the fit are down at the noise level. The integrated cross-sections of blended lines have been separated by using branching ratios observed for other transitions together with the Boltzmann population distribution. The integrated cross-sections of $R_{11}$ branch lines are plotted in Fig. 3. The lines with $J>4$ consist of two components, $e$ and $f$, indicated by solid and open circles, respectively. The total crosssections are represented by the solid squares. These crosssections are derived from the total column density of $\mathrm{NO}$ and therefore depend on the temperature, which was $295 \mathrm{~K}$ in this case. The lines belonging to the $P_{11}$ branch form a bandhead and are strongly overlapped, as indicated by the blending shown in Table I. Hence only the total integrated crosssection is given in Table III. The values listed in Table III can be divided by the fractional populations of the rotational levels to obtain values proportional to the line oscillator strengths. The strengths of the branches are obtained by adding the integrated cross-sections of all rotational lines in the branch. These are listed at the bottom line of Table III.

The uncertainties in the integrated cross-sections are due to noise and to errors in the measurement of the NO pressure and optical path length. Because the cross-sections from the same optical path length $0.565 \mathrm{~cm}$ with two different NO pressures coincided well, the error in the pressure measurements was considered small. However, cross-sections from the shorter path $0.284 \mathrm{~cm}$ deviated by as much as $40 \%$ from those with the longer path $0.565 \mathrm{~cm}$. The discrepancy is believed to be due to uncertainty in the length of the shorter path, and therefore the cross-sections from this path were not included in the calculation of the averaged value of the crosssections. The differences between the other two data sets with the same path length were compatible with the noise level. The photon noise in FT spectroscopy is virtually constant throughout the spectrum, and in our experiments it amounted typically to an uncertainty for a single point in the cross-section of $\pm 1.70 \times 10^{-18} \mathrm{~cm}^{2}$ for lines that are on the linear part of the curve of growth; the uncertainty for partly saturated lines is rather greater. The uncertainty for a strong unblended line of integrated cross-section 3 $\times 10^{-17} \mathrm{~cm}^{2} \mathrm{~cm}^{-1}$ is $5.6 \%$. For weak lines of integrated cross-section $2 \times 10^{-17}$ and $1 \times 10^{-17} \mathrm{~cm}^{2} \mathrm{~cm}^{-1}$ the uncertainties are $9 \%$ and $17 \%$, respectively. As the integrated band strength is determined predominantly by the strong lines, we ascribe an error of $6 \%$ to this. For all branches the integrated cross-sections were found to be a smooth function of $J$ which reflects the Boltzmann distribution in the ground state, but several unexpectedly large integrated cross-sections were obtained at $R_{22}(12.5)_{e}$ and $P_{21}(12.5)_{e}$. The reason for these large integrated cross-sections is not understood.

The band oscillator strength of $a\left(v^{\prime}, v^{\prime \prime}\right)$ band is given by 
TABLE I. Observed wavenumbers of the $\delta(1,0)$ band of NO. ${ }^{\text {a }}$

\begin{tabular}{|c|c|c|c|c|c|c|c|c|}
\hline$J$ & $R_{11}(J)_{e}$ & $R_{11}(J)_{f}$ & $R_{12}(J)_{e}$ & $R_{12}(J)_{f}$ & $R_{21}(J)_{e}$ & $R_{21}(J)_{f}$ & $R_{22}(J)_{e}$ & $R_{22}(J)_{f}$ \\
\hline 0.5 & \multicolumn{2}{|c|}{54 692.738B } & \multicolumn{6}{|c|}{54699.329} \\
\hline 1.5 & \multicolumn{2}{|c|}{54695.189} & \multicolumn{2}{|c|}{54 575.273B } & \multicolumn{2}{|c|}{$54706.084 \mathrm{~B}$} & \multicolumn{2}{|c|}{54586.216} \\
\hline 2.5 & \multicolumn{2}{|c|}{54698.319} & \multicolumn{2}{|c|}{$54578.206 \mathrm{~B}$} & 54713.469 & 54713.113 & 54593.359 & 54593.046 \\
\hline 3.5 & \multicolumn{2}{|c|}{54 701.994B } & \multicolumn{2}{|c|}{$54581.493 B$} & 54721.224 & $54720.721 B$ & 54600.750 & $54600.265 b$ \\
\hline 4.5 & $54706.084 \mathrm{~B}$ & 54706.522 & $54585.110 \mathrm{~B}$ & 54585.613 & 54729.511 & $54728.931 \mathrm{~B}$ & 54608.563 & 54607.900 \\
\hline 5.5 & 54710.818 & $54711.320 \mathrm{~B}$ & $54589.294 \mathrm{~b}$ & $54589.935 b$ & 54738.282 & 54737.324 & 54616.818 & 54 615.928B \\
\hline 6.5 & $54715.958 \mathrm{~B}$ & 54716.721 & 54593.886 & $54594.701 \mathrm{~B}$ & 54747.554 & 54746.348 & 54625.472 & $54624.400 \mathrm{~B}$ \\
\hline 7.5 & 54721.628 & 54722.619 & $54598.833 B$ & $54599.915 b$ & 54757.345 & 54755.840 & 54634.543 & 54633.141 \\
\hline 8.5 & 54727.749 & 54728.931 & 54604.163 & 54605.519 & $54767.455 \mathrm{~B}$ & 54765.772 & 54643.994 & $54642.290 \mathrm{~B}$ \\
\hline 9.5 & 54734.345 & 54735.896 & 54609.858 & 54611.506 & 54778.280 & 54776.135 & 54653.799 & 54651.749 \\
\hline 10.5 & 54741.355 & 54743.216 & $54615.928 B$ & 54617.871 & 54789.389 & 54786.862 & $54664.011 \mathrm{~B}$ & 54 661.474B \\
\hline 11.5 & 54748.746 & 54750.947 & $54624.400 \mathrm{~B}$ & $54624.400 \mathrm{~B}$ & 54800.787 & $54797.880 \mathrm{~B}$ & 54674.315 & $54671.536 \mathrm{~B}$ \\
\hline 12.5 & 54756.472 & 54759.030 & 54628.812 & 54631.501 & 54812.526 & 54809.231 & 54684.945 & 54681.720 \\
\hline 13.5 & 54764.438 & 54767.455 & 54635.567 & 54638.624 & 54824.324 & 54820.635 & $54695.567 \mathrm{~B}$ & 54691.904 \\
\hline 14.5 & 54772.499 & 54775.812 & $54642.290 \mathrm{~B}$ & 54645.774 & 54835.920 & 54831.923 & $54706.084 \mathrm{~B}$ & $54701.994 B$ \\
\hline 15.5 & 54780.441 & 54784.070 & 54652.694 & 54648.930 & 54846.955 & 54842.636 & 54715.506 & $54711.320 \mathrm{~B}$ \\
\hline 16.5 & 54787.823 & 54791.707 & 54654.783 & 54658.910 & & & & \\
\hline 17.5 & 54793.859 & 54797.880 & & & & & & \\
\hline 18.5 & 54797.079 & 54800.787 & & & & & & \\
\hline$J$ & $P_{11}(J)_{e}$ & $P_{11}(J)_{f}$ & $P_{12}(J)_{e}$ & $P_{12}(J)_{f}$ & $P_{21}(J)_{e}$ & $P_{21}(J)_{f}$ & $P_{22}(J)_{e}$ & $P_{22}(J)_{f}$ \\
\hline \multicolumn{9}{|l|}{0.5} \\
\hline 1.5 & & & & & & & & \\
\hline 2.5 & \multicolumn{2}{|c|}{54679.255} & \multicolumn{2}{|c|}{54559.107} & \multicolumn{2}{|c|}{$54685.969 \mathrm{~B}$} & \multicolumn{2}{|c|}{$54565.749 \mathrm{~B}$} \\
\hline 3.5 & \multicolumn{2}{|c|}{54675.104} & \multicolumn{2}{|c|}{54554.621} & \multicolumn{2}{|c|}{$54685.969 \mathrm{~B}$} & \multicolumn{2}{|c|}{$54565.749 \mathrm{~B}$} \\
\hline 4.5 & \multicolumn{2}{|c|}{$54671.536 \mathrm{~B}$} & \multirow{2}{*}{\multicolumn{2}{|c|}{$\begin{array}{l}54550.650 \\
54547.151\end{array}$}} & 54687.744 & 54687.275 & \multicolumn{2}{|c|}{$54565.749 \mathrm{~B}$} \\
\hline 5.5 & \multicolumn{2}{|c|}{$54688.636 \mathrm{~B}$} & & & 54687.780 & 54687.266 & $54566.353 b$ & $54565.749 \mathrm{~B}$ \\
\hline 6.5 & 54665.994 & 54666.340 & 54543.893 & 54544.340 & 54689.391 & 54688.639 & 54567.314 & $54566.660 \mathrm{~b}$ \\
\hline 7.5 & $54664.011 \mathrm{~B}$ & 54664.512 & 54541.186 & 54541.808 & 54691.482 & 54690.506 & 54568.679 & 54567.760 \\
\hline 8.5 & $54662.365 \mathrm{~B}$ & 54663.259 & 54538.884 & 54539.720 & 54694.089 & $54692.738 \mathrm{~B}$ & 54570.484 & 54569.347 \\
\hline 9.5 & $54661.474 \mathrm{~B}$ & $54662.365 \mathrm{~B}$ & $54536.972 b$ & 54538.076 & 54697.156 & $54695.567 \mathrm{~B}$ & 54572.686 & 54571.273 \\
\hline 10.5 & $54660.840 \mathrm{~B}$ & $54662.365 \mathrm{~B}$ & $54535.443 \mathrm{~B}$ & $54536.785 b$ & 54700.720 & 54698.893 & $54575.273 \mathrm{~B}$ & 54573.550 \\
\hline 11.5 & $54660.840 \mathrm{~B}$ & $54662.365 \mathrm{~B}$ & 54534.282 & $54535.937 \mathrm{~B}$ & 54704.726 & 54702.553 & $54578.206 \mathrm{~B}$ & 54576.172 \\
\hline 12.5 & $54661.047 \mathrm{~B}$ & $54662.979 \mathrm{~B}$ & 54533.480 & $54535.443 \mathrm{~B}$ & 54709.158 & $54706.084 \mathrm{~B}$ & $54581.493 \mathrm{~B}$ & 54579.096 \\
\hline 13.5 & 54662.016 & $54664.011 \mathrm{~B}$ & $54532.960 \mathrm{~b}$ & $54535.443 B$ & 54713.912 & $54711.320 \mathrm{~B}$ & $54585.110 \mathrm{~B}$ & 54582.275 \\
\hline 14.5 & $54662.979 \mathrm{~B}$ & $54665.459 \mathrm{~B}$ & $54532.622 B$ & $54535.443 B$ & 54718.919 & $54715.958 \mathrm{~B}$ & $54588.759 \mathrm{~b}$ & 54585.613 \\
\hline 15.5 & $54664.011 \mathrm{~B}$ & $54666.947 \mathrm{~B}$ & & & 54724.032 & $54720.721 \mathrm{~B}$ & $54592.516 \mathrm{~b}$ & $54588.973 b$ \\
\hline 16.5 & $54665.459 \mathrm{~B}$ & $54688.636 \mathrm{~B}$ & & & $54728.931 B$ & 54724.924 & 54595.965 & $54592.137 b$ \\
\hline 17.5 & $54666.947 \mathrm{~B}$ & 54670.412 & & & 54733.284 & $54728.931 \mathrm{~B}$ & $54598.833 B$ & $54594.701 B$ \\
\hline 18.5 & 54667.512 & & & & & & & \\
\hline & & $J$ & & $Q_{11}(J)$ & & $Q_{22}(J)$ & & \\
\hline & & 0.5 & & 54690.004 & & & & \\
\hline & & 1.5 & & & & 54575.420 & & \\
\hline & & 2.5 & & & & 54577.572 & & \\
\hline
\end{tabular}

${ }^{a}$ Lines followed by B are observed as a single line and by $b$ are observed as an incompletely resolved complex.

$$
f\left(\mathrm{v}^{\prime}, \mathrm{v}^{\prime \prime}\right)=\frac{m c^{2}}{\pi e^{2}} \frac{1}{\widetilde{N}\left(\mathrm{v}^{\prime \prime}\right)} \int \sigma(\nu) d \nu,
$$

in which $\widetilde{N}\left(\mathrm{v}^{\prime \prime}\right)$ is the Boltzmann population of the absorbing vibrational level, and the integration of the cross-section $\sigma(\nu)$ is performed over all of the rotational lines belonging to the $\left(\mathrm{v}^{\prime}, \mathrm{v}^{\prime \prime}\right)$ band. The total cross-sections of observed lines for each branch are presented in Table III. Observations of the rotational lines are mostly limited to $J \leqslant 16.5$. The effects from higher $J$ lines cannot be ignored. We extended the sum to $J=33.5$ by using the Boltzmann distribution and these value are presented in Table III as "Extended." Yoshino et al. ${ }^{32}$ made a similar correction for photoabsorption cross-section of Herzberg II bands of $\mathrm{O}_{2}$. For the $P_{11}$ branch the individual line cross-sections could not be measured because of the blending, so only the total is given in Table III. As the highest $J$-value included in the integration is not known, we have not attempted to estimate the effect of the high- $J$ lines on this total. In the present case the rotational levels corresponding to values of $J$ above 11.5 of $C^{2} \Pi(\mathrm{v}=1)$ are mixed with $B^{2} \Pi(\mathrm{v}=10),{ }^{26}$ and thus the mixing ratio must be taken into account to obtain the true band oscillator strength of the $\delta(1,0)$ band. This analysis will be presented after the analysis of the $\beta(10,0)$ band is completed.

Despite the mixing with the $B^{2} \Pi(\mathrm{v}=10)$ level, the perturbed oscillator strength of the $\delta(1,0)$ band can be compared with previous published values. The sum of the integrated 


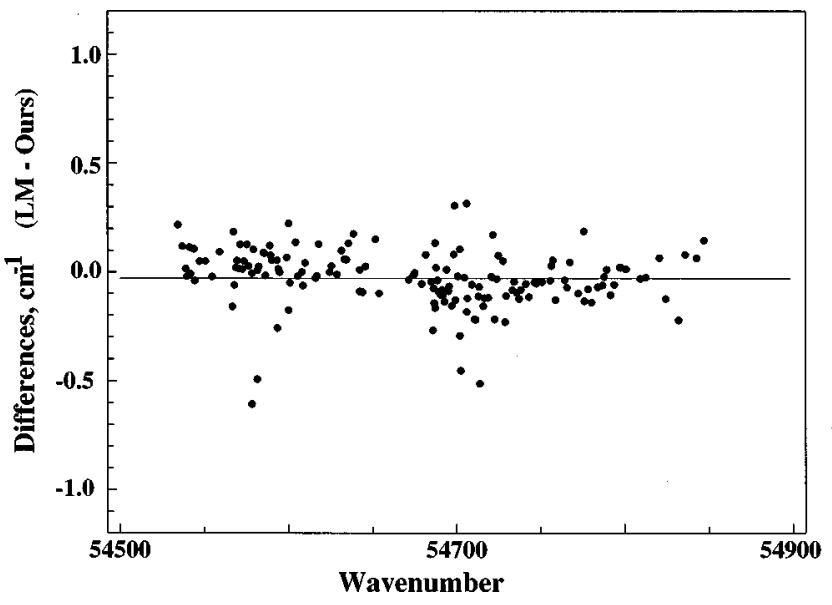

FIG. 2. Differences in the observed line positions between our results and Lagerqvist and Miescher (Ref. 26). The differences are given by the solid circles, and the solid horizontal line represents the average shift of $-0.03 \mathrm{~cm}^{-1}$.

cross-sections of all observed rotational lines in Table III is $4.79 \times 10^{-15} \mathrm{~cm}^{2} \mathrm{~cm}^{-1}$. The band oscillator strength $f(1,0)$ determined from the above integrated cross-section is $(5.4$ $\pm 0.3) \times 10^{-3}$. This value agrees reasonably well with the previous values of $6.0 \times 10^{-3}$ by Chan et al., ${ }^{22} 5.8 \times 10^{-3}$ by Bethke, ${ }^{7}$ and $5.6 \times 10^{-3}$ by Cieslik, ${ }^{8}$ but is $57 \%$ lower than the value of $8.5 \times 10^{-3}$ reported by Guest: ${ }^{9}$ the reason for this is not known. Our value is smaller than any of the previous values, but it is the only one derived from line-by-line measurements with a resolution comparable to the Doppler widths. Moreover, we were able to separate the lines of the

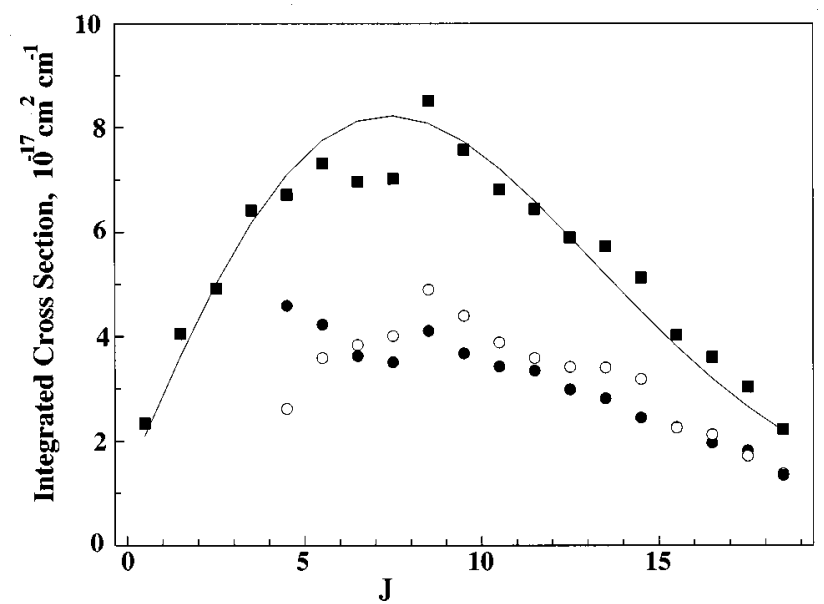

FIG. 3. The integrated cross-sections of the $R_{11}$ branch lines. The lines with $J>4$ consist of two components, $e$ and $f$, shown by solid and open circles, respectively. The total cross-sections are represented by solid squares.

$\delta(1,0)$ band from those of the overlapping $\beta(10,0)$ band. This could not be done in the previous low resolution measurements, and it is possible that the blends may have led to overestimates of the band oscillator strength for the $\delta(1,0)$ band by these authors. Bethke's value agrees with ours despite the possible error in his value pointed out by Callear and Pilling. ${ }^{13}$

\section{SUMMARY}

This work provides the first absorption measurements of the $\delta(1,0)$ band of NO free of problems arising from inad-

TABLE II. The term values and molecular constants of $C(1){ }^{2} \Pi$ level of NO, $\mathrm{cm}^{-1}$.

\begin{tabular}{|c|c|c|c|c|}
\hline \multirow[b]{2}{*}{$J$} & \multicolumn{2}{|c|}{$F_{1},{ }^{2} \Pi_{1 / 2}$} & \multicolumn{2}{|c|}{$F_{2},{ }^{2} \Pi_{3 / 2}$} \\
\hline & $e$ & $f$ & $e$ & $f$ \\
\hline 0.5 & $54690.000 \pm 0.009$ & $54690.012 \pm 0.009$ & & \\
\hline 1.5 & $54692.611 \pm 0.013$ & $54692.629 \pm 0.027$ & $54699.329 \pm 0.016$ & $54699.353 \pm 0.016$ \\
\hline 2.5 & $54700.176 \pm 0.014$ & $54700.193 \pm 0.022$ & $54711.124 \pm 0.006$ & $54711.120 \pm 0.006$ \\
\hline 3.5 & $54711.664 \pm 0.020$ & $54711.702 \pm 0.008$ & $54726.852 \pm 0.019$ & $54726.538 \pm 0.019$ \\
\hline 4.5 & $54727.068 \pm 0.024$ & $54727.084 \pm 0.028$ & $54746.280 \pm 0.009$ & $54745.819 \pm 0.009$ \\
\hline 5.5 & $54746.192 \pm 0.015$ & $54746.656 \pm 0.020$ & $54769.602 \pm 0.006$ & $54768.939 \pm 0.006$ \\
\hline 6.5 & $54769.241 \pm 0.116$ & $54769.883 \pm 0.010$ & $54796.766 \pm 0.010$ & $54795.878 \pm 0.010$ \\
\hline 7.5 & $54796.183 \pm 0.011$ & $54797.021 \pm 0.005$ & $54827.781 \pm 0.006$ & $54826.643 \pm 0.006$ \\
\hline 8.5 & $54826.916 \pm 0.006$ & $54828.001 \pm 0.003$ & $54862.628 \pm 0.007$ & $54861.219 \pm 0.007$ \\
\hline 9.5 & $54861.450 \pm 0.011$ & $54862.818 \pm 0.008$ & $54901.289 \pm 0.006$ & $54899.582 \pm 0.006$ \\
\hline 10.5 & $54899.811 \pm 0.013$ & $54901.473 \pm 0.009$ & $54943.740 \pm 0.009$ & $54941.707 \pm 0.009$ \\
\hline 11.5 & $54941.933 \pm 0.015$ & $54943.907 \pm 0.003$ & $54989.955 \pm 0.008$ & $54987.556 \pm 0.008$ \\
\hline 12.5 & $54987.759 \pm 0.008$ & $54990.094 \pm 0.006$ & $55039.842 \pm 0.007$ & $55037.077 \pm 0.007$ \\
\hline 13.5 & $55037.260 \pm 0.014$ & $55039.975 \pm 0.006$ & $55093.320 \pm 0.005$ & $55090.180 \pm 0.005$ \\
\hline 14.5 & $55090.360 \pm 0.013$ & $55093.439 \pm 0.015$ & $55150.254 \pm 0.015$ & $55146.730 \pm 0.015$ \\
\hline 15.5 & $55146.904 \pm 0.005$ & $55150.380 \pm 0.016$ & $55210.306 \pm 0.018$ & $55206.484 \pm 0.018$ \\
\hline 16.5 & $55206.647 \pm 0.013$ & $55210.461 \pm 0.010$ & $55273.196 \pm 0.029$ & $55269.042 \pm 0.029$ \\
\hline 17.5 & $55269.155 \pm 0.050$ & $55273.245 \pm 0.038$ & & \\
\hline \multirow[t]{8}{*}{18.5} & $55333.718 \pm 0.006$ & $55337.878 \pm 0.005$ & & \\
\hline & $T_{0}$ & 54690.155 & \pm 0.017 & \\
\hline & $A$ & 3.556 & \pm 0.078 & \\
\hline & $B_{\mathrm{v}}$ & 1.94406 & \pm 0.00062 & \\
\hline & $D_{\mathrm{v}}, 10^{-5}$ & 5.91 & \pm 0.42 & \\
\hline & $A_{D}$ & -0.0187 & \pm 0.0050 & \\
\hline & $p$ & -0.0189 & \pm 0.0037 & \\
\hline & $q$ & -0.01521 & \pm 0.00020 & \\
\hline
\end{tabular}


TABLE III. Integrated cross sections of lines of the $\delta(1,0)$ band of NO in units of $10^{-17} \mathrm{~cm}^{2} \mathrm{~cm}^{-1}$.

\begin{tabular}{|c|c|c|c|c|c|c|c|c|c|}
\hline \multirow[b]{2}{*}{$J$} & \multicolumn{3}{|c|}{$R_{11}(J)$} & \multicolumn{2}{|l|}{$R_{12}(J)$} & \multicolumn{2}{|c|}{$R_{21}(J)$} & \multicolumn{2}{|c|}{$R_{22}(J)$} \\
\hline & $e$ & $f$ & & $e$ & $f$ & $e$ & $f$ & $e$ & $f$ \\
\hline 0.5 & \multicolumn{3}{|c|}{$1.79 \mathrm{~B}$} & & & \multicolumn{2}{|c|}{1.52} & & \\
\hline 1.5 & \multicolumn{3}{|c|}{3.52} & \multicolumn{2}{|l|}{$1.31 \mathrm{~B}$} & \multicolumn{2}{|c|}{$2.77 \mathrm{~B}$} & \multicolumn{2}{|c|}{1.22} \\
\hline 2.5 & \multirow{2}{*}{\multicolumn{3}{|c|}{$\begin{array}{l}4.47 \\
6.28 \mathrm{~B}\end{array}$}} & \multicolumn{2}{|l|}{$2.08 \mathrm{~B}$} & 1.91 & 1.64 & 0.58 & 1.33 \\
\hline 3.5 & & & & \multicolumn{2}{|l|}{$2.78 \mathrm{~B}$} & 2.88 & $1.54 \mathrm{~B}$ & $1.06 \mathrm{~b}$ & $2.17 \mathrm{~b}$ \\
\hline 4.5 & 3.96B & \multirow{2}{*}{\multicolumn{2}{|c|}{$\begin{array}{l}2.32 \\
3.23 \mathrm{~B}\end{array}$}} & $1.90 \mathrm{~B}$ & 1.42 & 1.65 & $2.13 \mathrm{~B}$ & 1.82 & 1.63 \\
\hline 5.5 & 3.65 & & & $1.44 \mathrm{~b}$ & 25 & 2.35 & 2.29 & 1.97 & $1.75 \mathrm{~B}$ \\
\hline 6.5 & 3.17B & 3.6 & & 1.68 & 67B & 2.48 & 2.60 & 2.07 & $2.25 \mathrm{~B}$ \\
\hline 7.5 & 3.14 & 3.62 & & $1.31 \mathrm{~B}$ & 09b & 2.51 & 2.12 & 2.21 & 1.97 \\
\hline 8.5 & 3.70 & 4.58 & & 1.24 & 52 & $2.71 \mathrm{~B}$ & 2.04 & 2.13 & $1.86 \mathrm{~B}$ \\
\hline 9.5 & 3.29 & 3.95 & & 1.02 & 46 & 2.00 & 1.67 & 2.10 & 2.09 \\
\hline 10.5 & 3.08 & 3.40 & & $1.00 \mathrm{~B}$ & 25 & 1.90 & 1.75 & & \\
\hline 11.5 & 2.87 & 3.23 & & 0.79 & 89B & 1.88 & $2.16 \mathrm{~B}$ & 1.76 & $1.18 \mathrm{~B}$ \\
\hline 12.5 & 2.68 & 2.9 & & 0.82 & 81 & 1.48 & 1.33 & 4.54 & 1.63 \\
\hline 13.5 & 2.29 & 2.95 & & 0.51 & 70 & 1.40 & 1.01 & $1.40 \mathrm{~B}$ & 1.31 \\
\hline 14.5 & 2.17 & 2.5 & & $0.52 \mathrm{~B}$ & 68 & 1.31 & 0.86 & $1.19 \mathrm{~B}$ & $1.33 \mathrm{~B}$ \\
\hline 15.5 & 1.79 & 1.8 & & 0.31 & 43 & 0.90 & 0.71 & 1.24 & $0.89 \mathrm{~B}$ \\
\hline 16.5 & 1.52 & 1.68 & & 0.73 & 09 & & & & \\
\hline 17.5 & 1.36 & 1.27 & & & & & & & \\
\hline 18.5 & 1.79 & 0.57 & & & & & & & \\
\hline Total $^{\mathrm{b}}$ & \multicolumn{3}{|c|}{98.30} & \multicolumn{2}{|l|}{33.71} & 55.48 & & 45.8 & \\
\hline \multirow[t]{2}{*}{ Extended $^{\mathrm{c}}$} & 103 & & & 36.71 & & 62.52 & & 51.4 & \\
\hline & $P_{11}(J)$ & $P_{12}$ & & & $(J)$ & & ${ }_{2}(J)$ & $Q_{11}(J)$ & $Q_{22}(\mathrm{~J})$ \\
\hline$J$ & $e, f$ & $e$ & $f$ & $e$ & $f$ & $e$ & $f$ & & \\
\hline 0.5 & & & & & & & & 1.35 & \\
\hline 1.5 & & & & & & & & & 1.79 \\
\hline 2.5 & & 0. & & & 48B & & 34 & & 1.16 \\
\hline 3.5 & & 1. & & & 33B & & 96 & & \\
\hline 4.5 & & 1. & & 1.77 & 3.62 & & 42 & & \\
\hline 5.5 & & 2. & & 2.75 & 2.04 & 1.73 & 1.88 & & \\
\hline 6.5 & & 0.99 & 1.02 & 2.29 & 2.10 & 2.40 & 1.50 & & \\
\hline 7.5 & & 1.06 & 1.11 & 2.29 & 1.93 & 3.42 & 1.96 & & \\
\hline 8.5 & & 1.07 & 1.38 & 2.39 & $2.47 \mathrm{~B}$ & 1.79 & 2.27 & & \\
\hline 9.5 & & $1.08 \mathrm{~b}$ & 1.10 & 2.04 & $1.79 \mathrm{~B}$ & 1.83 & 1.98 & & \\
\hline 10.5 & & $0.94 \mathrm{~B}$ & $0.92 b$ & 1.75 & 1.58 & $0.90 \mathrm{~B}$ & 1.69 & & \\
\hline 11.5 & & 1.34 & $0.63 \mathrm{~B}$ & 1.41 & 1.11 & $0.89 \mathrm{~B}$ & 1.71 & & \\
\hline 12.5 & & $0.67 b$ & $0.73 B$ & 1.54 & $1.86 \mathrm{~B}$ & $0.83 \mathrm{~B}$ & 1.46 & & \\
\hline 13.5 & & $0.77 b$ & $0.55 b$ & 0.87 & $0.71 \mathrm{~B}$ & $0.96 \mathrm{~B}$ & 1.15 & & \\
\hline 14.5 & & $0.72 \mathrm{~B}$ & $0.54 \mathrm{~B}$ & 0.59 & $0.55 \mathrm{~B}$ & $1.69 \mathrm{~b}$ & 0.59 & & \\
\hline 15.5 & & $0.59 \mathrm{~B}$ & $0.71 b$ & 0.64 & $1.29 \mathrm{~B}$ & $1.04 \mathrm{~b}$ & $1.21 \mathrm{~b}$ & & \\
\hline 16.5 & & $0.49 \mathrm{~B}$ & $0.27 \mathrm{~B}$ & $0.72 B$ & 1.01 & 1.27 & $1.42 \mathrm{~b}$ & & \\
\hline 17.5 & & & & 0.29 & $0.57 \mathrm{~B}$ & 0.30 & $0.36 \mathrm{~B}$ & & \\
\hline Total $^{\mathrm{b}}$ & 90.84 & 26. & & 49 & & & & 1.35 & 2.95 \\
\hline Extended $^{\mathrm{c}}$ & & 28. & & 52 & & & & & \\
\hline
\end{tabular}

${ }^{a}$ Lines followed by $B$ are observed as a single line and by $b$ are observed as an incompletely resolved complex.

${ }^{\mathrm{b}}$ Total cross section for observed lines.

${ }^{\mathrm{c}}$ Total cross section after correction of higher $J$ lines which are not observed. See text for detail.

equate spectral resolution, achieved by using the combination of a VUV FT spectrometer and a synchrotron radiation source. Accurate rotational line positions and term values as well as the photoabsorption integrated cross-sections are provided.

\section{ACKNOWLEDGMENTS}

This work was supported in part by a NSF Division of Atmospheric Sciences Grant No. ATM-94-22854 to Harvard College Observatory, and by the NASA Upper Atmospheric Research Program under Grant No. NAG5-484 to the Smithsonian Astrophysical Observatory. We also acknowledge the
Paul Instrument Fund of the Royal Society for the development of the VUV-FT spectrometer. The FTS measurements at the Photon factory were made with the approval of the Photon Factory Advisory Committee (94G367). K.Y. thanks the Japan Society for the Promotion of Science for support.

${ }^{1}$ G. Brasseur and S. Solomon, Aeronomy of the Middle Atmosphere (Reidel, New York, 1984).

${ }^{2}$ T. Shimazaki, Minor Constituents in the Middle Atmosphere (Terra Scientific, Tokyo, 1985).

${ }^{3}$ M. Nicolet, Ann. Geophys. (France) 1, 493 (1983).

${ }^{4}$ J. E. Frederic, A. J. Blake, and D. E. Freeman, Handbook for MAP 8 (53 ICSC Scientific Committee on Solar Terrestrial Physics, Urbana, IL, 1983). 
${ }^{5}$ M. Nicolet and R. Kennis, Planet. Space Sci. 37, 457 (1989).

${ }^{6}$ K. Minschwaner, R. J. Salawitch, and M. B. McElroy, J. Geophys. Res. 98, 10543 (1993).

${ }^{7}$ G. W. Bethke, J. Chem. Phys. 31, 662 (1959).

${ }^{8}$ S. Cieslik, Bull. Cl. Sci., Acad. R. Belg. 63, 884 (1977).

${ }^{9}$ J. A. Guest and L. C. Lee, J. Phys. B 14, 3401 (1981).

${ }^{10}$ R. D. Hudson, Rev. Geophys. Space Phys. 9, 305 (1971).

${ }^{11}$ J. E. Murray, K. Yoshino, J. R. Esmond, W. H. Parkinson, Y. Sun, A. Dalgarno, A. P. Thorne, and G. Cox, J. Chem. Phys. 101, 62 (1994).

${ }^{12}$ K. Yoshino, J. R. Esmond, W. H. Parkinson, A. P. Thorne, J. E. Murray, R. C. M. Learner, G. Cox, A. S.-C. Cheung, K. W.-S. Leung, K. Ito, T. Matsui, and T. Imajo, J. Chem. Phys. 109, 1751 (1998).

${ }^{13}$ A. B. Callear and M. J. Pilling, Trans. Faraday Soc. 66, 1886 (1970).

${ }^{14}$ T. W. Dingle, P. A. Freedman, B. Gelernt, W. J. Jones, and I. W. M. Smith, Chem. Phys. 8, 171 (1975).

${ }^{15}$ O. Benoist D’Azy, R. Lopez-Delgado, and A. Tramer, Chem. Phys. 9, 327 (1975).

${ }^{16}$ K. Tsukiyama, T. Munakata, M. Tsukakoshi, and T. Kasuya, Chem. Phys. 137, 315 (1987).

${ }^{17}$ K. Tsukiyama, T. Munakata, M. Tsukakoshi, and T. Kasuya, Chem. Phys. 121, 55 (1988).

${ }^{18}$ T. Hikida, T. Suzuki, and Y. Moru, Chem. Phys. 118, 437 (1987).
${ }^{19}$ D. J. Hart and O. L. Bourne, Chem. Phys. 133, 103 (1989).

${ }^{20}$ C. Amiot and J. Verges, Phys. Scr. 25, 302 (1982).

${ }^{21} \mathrm{C}$. Amiot (private communication).

${ }^{22}$ W. F. Chan, G. Cooper, and C. E. Brion, Chem. Phys. 170, 111 (1993).

${ }^{23}$ W. H. Parkinson, A. P. Thorne, G. Cox, P. L. Smith, and K. Yoshino, Proc. SPIE 2282, 58 (1994).

${ }^{24}$ J. W. Brault (private communication).

${ }^{25}$ P. M. Dooley, B. R. Lewis, S. T. Gibson, G. H. Baldwin, P. C. Cosby, J. L. Price, R. A. Copeland, T. G. Slanger, K. Yoshino, A. P. Thorne, and J. E. Murray, J. Chem. Phys. 109, 3856 (1998).

${ }^{26}$ A. Lagerqvist and E. Miescher, Helv. Phys. Acta 31, 221 (1958).

${ }^{27}$ C. Amiot, R. Bacis, and G. Guelachvili, Can. J. Phys. 56, 251 (1978).

${ }^{28}$ J. T. Hougen, NBS Monograph 115 (U.S. Government Printing Office, Washington, DC., 1970).

${ }^{29}$ R. N. Zare, A. L. Schmeltekopf, D. L. Albritton, and W. J. Harrop, J. Mol. Spectrosc. 48, 174 (1973).

${ }^{30}$ C. Amiot, J-P. Maillard, and J. Chauville, J. Mol. Spectrosc. 87, 196 (1981).

${ }^{31}$ G. Stark, J. W. Brault, and M. C. Abrams, J. Opt. Soc. Am. B 11, 3 (1994).

${ }^{32}$ K. Yoshino, J. R. Esmond, W. H. Parkinson, A. P. Thorne, R. C. M. Learner, and G. Cox, J. Chem. Phys. 111, 2960 (1999). 